\title{
Peter Lenhart, Marianne Schuller, Jasmin Sohnemann \& Manuel Zahn (Hrsg.): Wo ist das Über-Ich und was macht es dort? Studien zu einem psychoanalytischen Begriff (Parodos, Berlin, 2014) Olaf Knellessen (Zürich)
}

«Die Rede vom Über-Ich ist so geläufig wie dunkel. Das Über-Ich wandert ebenso unbehelligt und leicht geschürzt durch Alltagsdiskurse wie es als psychoanalytischer Begriff schwer zu fassen ist» (Lenhart et al. [Hrsg.], 2014, S. 7). So beginnt das Buch Wo ist das Über-Ich und was macht es dort, der Hamburger Forschungsgruppe für Psychoanalyse. Schon der Titel stellt die Frage nach der Topologie, die zudem durch seine Gestaltung auf dem Cover unterstrichen wird. Das Über-Ich ist zentriert und vertikal nach oben ausgerichtet - sozusagen auf den Kopf gestellt -, wobei ihm oben links die Frage Wo ist das voran, unten rechts dann die weitere Frage und was macht es dort? nachgestellt wird.

Sehr unbehelligt dürfte es in dieser räumlichen Position zwischen oben und unten, zwischen links und rechts allerdings nicht sein. Aber es steht doch sehr aufrecht da und vielleicht passt dies zur leisen Frivolität des Leicht-Geschürzten ebenso wie zur Schwere und zur bekannten Strenge, die es häufig auszeichnet, dieses Über-Ich. So verweist auch die Bedeutung der Stimme, die von Mladen Dolar (2007) im bekannten Bild des Schallplatten-Labels von His Master's Voice dem Über-Ich zugeschrieben wird, ja eben auch nicht nur auf die Strenge, sondern auch auf die Musik, auf den Gesang und auf den Tanz.

Die spezifische Räumlichkeit des Über-Ichs wird schon im ersten Beitrag von Julian Rohrhuber entfaltet. Er geht aus vom Traum über einen verlorenen Gegenstand, der einen plagt und quält. Man möchte ihn wieder finden und begibt sich auf der Suche nach ihm auf einen ständigen Weg zurück, während der Traum gleichzeitig weiter und weiter geht. Ein solcher Traum beschreibt, so Rohrhuber, das Bild einer Reuse, des Fangnetzes der Fischer. Ohne es zu bemerken, gerät man in dieses Netz, treibt weiter und weiter, es gibt kein zurück, und erreicht doch nie das Verlorene, das Gesuchte. Das anfängliche Treiben-Lassen verliert seine Leichtigkeit und Frivolität und wird zum Gefängnis, das wäre die Situation. 
Gleichzeitig wird eine andere Topologie des Über-Ichs entworfen als die vertikale, die über dem Ich steht. In dieser scheint es um ein «zwischen» zu gehen, um ein Verhältnis zwischen Innen und Aussen, in dem das Über-Ich sich situiert und wirkt. Und der Raum, in dem es sich bewegt, ist nicht einer, der frei verfügbar und vermessbar wäre, sondern einer, in dem der gesuchte Ort nicht zu finden ist, sondern unerfüllbare Anforderung bleibt. Ein fulminanter Auftakt.

Je ungewisser das Gelände umso bedeutsamer vielleicht die Aufrichte. Die Aufrichtung des Über-Ichs ist dem Fehlen einer konsistenten Ordnung zu verdanken (Adrienne Cromelin) und wie aufrecht dieses Über-Ich dann dastehen kann, sieht man auf dem Cover. In seiner Dimension des «Über» bietet es Ausblick und Übersicht. Eine solche Übersicht über den Begriff selbst, den Freud erst 1923 in Das Ich und das Es prägte, bietet der Beitrag von Claus-Dieter Rath. Im Über-Ich spiegelt sich, so könnte man mit ihm sagen, die Widersprüchlichkeit, in der das Subjekt steht. Mit Freud vertritt es «Tradition und Idealbildung der Vergangenheit» und leistet von daher selbst neuen Situationen eine Zeit lang «Widerstand) (Freud, 1932, S. 194)» (zit. nach Rath in: Lenhart et al. [Hrsg.], 2014, S. 113).

Gerade als Niederschlag der Identifikation mit der Elterninstanz - wie Freud es nannte -, und eben nicht mit den Personen, kann das Über-Ich klare, unmissverständliche Worte sprechen. Dann wird es zu einem, das bestimmt und definiert, sich hierarchisch darüber stellt. His master's voice ist auch in diesem Buch immer wieder zu vernehmen, wird bisweilen Lacan, bisweilen auch anderen zugeschrieben und zugemutet. Da kann der Blick auf unsere Welt sehr streng und sehr scharf werden - allerdings nicht mehr im Sinne von frivol.

Mit Referenz auf eine solche Stimme heisst es zum Beispiel: «Er sieht vielmehr in der gegenwärtigen Entwicklung einer global operierenden, digitalen hyperkulturellen Psychomacht und ihrer Zersetzung von individueller wie sozialer Aufmerksamkeit die Grundlagen für eine von Freud beschriebene sprimäre Identifizierung> zwischen den singulären Individuen eines Kollektivs gefährdet» (ebd., S. 148). Dann scheint, wie es an anderer Stelle heisst, die Befreiung verpasst, der aufrechte Gang, so könnte man sagen, gefährdet. So ungeliebt das Über-Ich auch immer wieder sein mag, so gerne man es manchmal los werden möchte, so bedrohlich scheint der immer wieder beschworene Eindruck, dass es beinahe schon abgeschafft ist und unsere Kultur damit nicht nur dem Niedergang, sondern dem Untergang geweiht ist. Das ist natürlich die vollkommen falsche Richtung für das Über-Ich.

Plötzlich diese Übersichthiess eine Ausstellung der Zürcher Künstler Fischli/ Weiss. In ihr vollzogen sie anfangs der 80er Jahre eine Wende, weg von einer Innerlichkeit, wie sie in den Jahren zuvor in der Kunst zelebriert wurde. Und sie 
vollzogen diese Wende - wie es der Titel schon sagt - mit Humor. Humor ist, wie Masaaki Sato mit Freud ausführt, «der Beitrag zur Komik durch die Vermittlung des Über-Ichs» (ebd., S. 76) und «macht den vorhandenen Sadismus unsadistisch geniessbar» (S. 77). Die Übersicht, die zum Über-Ich zu gehören scheint, muss also nicht nur schwer und streng sein, sie kann auch lachen, sie kann auch schmunzeln lassen - über die eigene Unzulänglichkeit und die der Welt.

Karl-Josef Pazzini erzählt die einzige Fallvignette in dem Buch, was nicht als Vorhaltung gemeint, sondern sehr wohltuend ist angesichts des Überhangs seröser Beispiele von Gelingen, der neuerdings die Psychoanalyse heimzusuchen scheint. Er hört sich gleich zu Beginn einer Stunde in einer mühsamen Phase zu seinem Analysanden sagen: « ¿Erzählen Sie mir von Ihren neuesten Verbrechen!〉 Das ging so schnell, dass ich diesen Satz gar nicht bedenken konnte. Das war Präsenz. Der Akt war ein Ereignis. Gleichzeitig mit diesem Schrecken, einem vom Über-Ich und Ichideal gesteuerten, erschrecke ich über ein explodierend lautes Lachen des Analysanden, derart laut und irre, dass ich intuitiv hochgucke, um zu kontrollieren, ob die Fenster geschlossen sind» (ebd., S. 101).

Auch wenn er sich versichert, dass die Fenster geschlossen sind: Da gehen die Fenster auf, plötzlich diese Übersicht. Gerade in dem Moment als etwas gesagt wurde, das eben keine Wiedergabe von etwas anderem war, keine Repräsentation, sondern Präsenz, in diesem Moment passiert es, die Fenster gehen auf. Und der Analysand «lacht Tränen. Und sagt: «Das ist es, genau das ist es: Ich habe nichts verbrochen, ich habe viele Verbrechen begangen, die letzten Tage»» (ebd., S. 102). Mit den Verbrechen wird eine andere Dimension des Über-Ichs ins Auge gefasst. Die des Übertritts, die des Überschreitens. Es befreit sich aus dem Fangnetz - auch hier mit Lachen und mit Humor - und überschreitet sich, geht über sich hinaus. Über-Ich wird damit nicht nur zu einer Richtung, die aufrecht steht, uns den aufrechten Gang ermöglicht, hierarchisch von oben nach unten blickt und sich auch über die Sexualität erhebt. Über-Ich wird zu einer Bewegung, die über sich, über das Ich hinaus geht, die Fenster aufmacht.

Die Gestaltung des Covers könnte man dann auch so sehen, dass dieses Über-Ich nicht nur aufrecht und dazwischen steht, zwischen oben und unten und links und rechts, dass es vielmehr dabei ist, die Frage nach seinem Ort aus sich herauszutreiben, ebenso wie die nach dem, was es tut. Das wäre nicht nur eine Topologie, sondern eine Dynamik, eine Bewegung, wie sie auch in den Beiträgen von Marianne Schuller und Renate Wieser gezeichnet und skizziert wird. Aby Warburgs «Nachleben» stellt nicht nur die Linearität von Zeit auf den Kopf - wie Freuds Nachträglichkeit -, sondern betont die Grenzenlosigkeit, die sich nicht so 
fesseln lässt wie der Laokoon. Und wenn «das Über-Ich als ein Exzess der Stimme, die als Überschreitung des Gesetzes fungiert» (Dollar, 2007, S. 131, zit.n. Wieser, in: Lenhart et al. [Hrsg.], 2014, S. 222), beschrieben wird und dies mit dem Hörspiel in Verbindung gebracht wird, bei dem die auftretenden Stimmen «immer sowohl als Stimmen im Raum oder Stimmen im Kopf verstanden werden» (ebd., S. 221), dann geht es auch hier um eine solche Überschreitung der Grenze zwischen Innen und Aussen, bei der das Innen sich ins Aussen überschreitet und von diesem Aussen ganz umgeben und umfangen wird. Da erhält His Master's Voice das wieder zurück, was zu diesem Label ja gehört: Es ist nicht einfach die strenge und befehlende Stimme, die von oben kommt, sie ist Musik, in die man eingetaucht ist, die einen umgibt.

Das vorliegende Buch bewegt sich in der Tat so durch das Feld des ÜberIchs, wie gleich am Anfang angekündigt: schwer und leicht geschürzt, mit düsteren Sichten, aber auch tanzend und lachend.

Dass es mit einem Beitrag von Ulrike Oudée Dünkelsbühler «Schreib dich!» schreit das Über-Ich: Lengage der Libellen endet, stimmt hoffnungsvoll - für das Über-Ich und für die Psychoanalyse. Der Text ist ein Kunstwerk. Er beginnt mit Derrida. «Ich muss Ihnen beibringen, mir beizubringen, mich zu lesen.» (ebd., S. 228) und geht weiter zu Helene Cixous: «Ecris-to! s'écrie-t-elle.» Und damit beginnt ein Feuerwerk, das die Sprache hört und über sich selbst hinaus treibt. Es heisst an einer Stelle, an vielen Stellen: «Ihr Text ist Einsatz, Wette, Pfand, das heisst gage, und es ist einer, der nicht konvertierend deutet, belehrt und bekehrt, sondern den Körper erschreibend gebiert, Hebamme von sich. Aber was heisst 〈sich»?» (ebd., S. 231).

Das Fliegen spielt eine grosse Rolle dabei, weil es ja auch ein Sich-über-sichhinaus-heben ist. Deshalb geht es auch um die Libelle, die mit und nach diesem Text nur noch so zu schreiben wäre: Lieb-elle!

Man kann sich nur noch bedanken für dieses Geschenk.

\section{Literatur}

Dollar, M. (2007).His Master's Voice. Eine Theorie der Stimme. Frankfurt a. M.: Suhrkamp.

Freud, S. (1932). Neue Folge der Vorlesungen zur Einführung in die Psychoanalyse. GW XV, Frankfurt a. M.: Fischer, 1969.

Lenhart, P., Schuller, M., Sohnemann, J. \& Zahn, M. (Hrsg.). (2014). Wo ist das ÜberIch und was macht es dort. Studien zu einem psychoanalytischen Begriff. Berlin: Parodos. 